\title{
BLENDERS FOR A NON-NORMALLY HÉNON-LIKE FAMILY
}

\author{
SHIN KIRIKI AND MASAKI NAKAJIMA
}

\begin{abstract}
A blender is an indispensable concept presented by Bonatti and Díaz [3] to study high-dimensional $C^{1}$-robust transitive dynamics around heterodimensional cycles. In this paper, we present a certain Hénon-like family of real quadratic diffeomorphisms on $\mathbb{R}^{3}$, which exhibits an phase transition from non-normal horseshoes to blenders. It can be observable from a rapidly jump of topological dimension for some projected stable segments in some characteristic region of $\mathbb{R}^{3}$.
\end{abstract}

\section{Introduction}

\subsection{Non-normally Hénon-like family}

A family of real quadratic polynomials

$$
f_{a, b}(x, y)=\left(1-a x^{2}+b y, x\right)
$$

or its topological conjugacy class is called a Hénon family, presented in [15]. Under appropriate conditions for parameters $(a, b)$, the family exhibits representative dynamical features from horseshoe structures to strange attractors via homoclinic bifurcations, see $[1,2,13,14]$. Moreover, the family plays a significant role in more general situations of two-dimensional dynamics near homoclinic tangencies, see in $[5,17,18]$. On the other hand, in higher than three-dimension, although several excellent results exist by using methods extended directly from two-dimensional cases as in [19,22], certain new mechanisms different from the two-dimensional cases are required to explain some phenomena such as bifurcations on heterodimensional cycles studied by Díaz and others $[3,8,9,10,11,12]$. A blender is indeed an essential concept to understand these results, first presented by Bonatti and Díaz [3, 5, 7]. Especially, it makes a significant contribution to obtain robustness of cycles close to a given heterodimensional bifurcation in the $C^{1}$ topology [4]. A precise definition of blender will be present in the next section.

Affine examples of blenders are already presented in $[5, \S 6.2 .1]$ and $[7, \S 3]$, which are constructed geometrically by adding three-dimensional deviations to non-normally affine

Corresponding author: Shin Kiriki.

Received March 11, 2009.

2000 Mathematics Subject Classification. Primary:37C29, 37D20, 37D30.

Key words and phrases. Robust transitive dynamics, heterodimensional cycles, Hénon-like family, non-normal horseshoes, blender. 
horseshoe maps on $\mathbb{R}^{3}$, see the next section. However, as far as authors know, there is no example which is defined by a real quadratic polynomial, like the Hénon family, which has blender structures. So, we here present a simple family of real polynomials on $\mathbb{R}^{3}$ defined as follows:

$$
\varphi_{a, b, c, d}(x, y, z)=\left(1-a x^{2}+b y, x, c z+d x\right) .
$$

Especially, for $|c|>1$, a topological conjugacy class of this family is called to be nonnormally Hénon-like in this paper.

Before stating the main result, let us give a brief overview for the family: If $|c|<1$ and $d=0$, since $\varphi_{a, b, c, d}(x, y, z)=\left(f_{a, b}(x, y), c z\right)$ is the essentially same as a normally Hénon-like family which are studied in $[22,6,21,16]$, we can not expect to detect blenderlike structures in such cases. Hence, we should focus on the family for $|c|>1$. Under the condition $d=0$, if $a$ and $b$ satisfy similar open conditions given by Devaney-Nitecki [13], $\varphi$ has a basic set, called a non-normally hyperbolic horseshoe in [7, §1], such that (i) the dimension of unstable bundle of basic set is equal to 2; (ii) the restriction for $\varphi$ to the basic set is conjugate to the full shift on two symbols; (iii) the basic set as well as it's stable and strong unstable manifolds are all embedded to the $x y$-plane (see Figure 1). That is, the nontrivial dynamics of $\varphi_{a, b, c, d}$ with $d=0$ is no different from that of two-dimensional Hénon family. Then, in this paper, we study what happens in three-dimensional dynamics of this family when the value of $d$ varies from 0 . In fact, one will observe that the parameter $d$ controls a structural deviation from nonnormally hyperbolic horseshoe to generate certain distinctive properties with respect to its invariant manifolds.

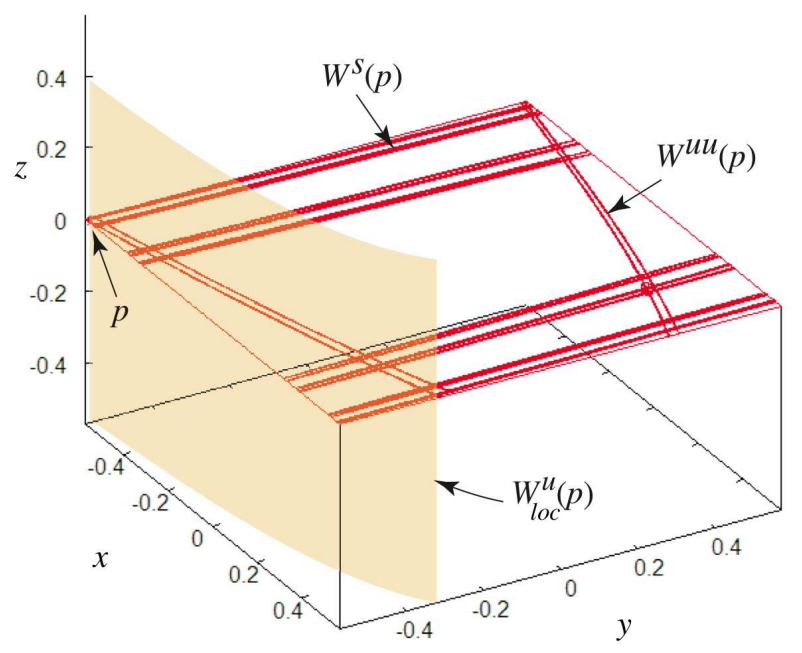

Figure 1: Stable, strong unstable segments and local unstable manifold of $p$ in the nonnormally hyperbolic horseshoe for $\varphi_{a, b, c, 0}$ for $a=5, b=-0.1$ and $c=1.1$. 
In the end of the section, we introduce one of advantages of our settings: the above polynomial definition allows us to simulate it on computers. For example, stable, strong unstable segments and local unstable manifold for some fixed point in the non-normally hyperbolic horseshoe for $\varphi_{a, b, c, d}$ with $d=0$ can be observed numerically as in Figure 1. In addition, several numerical pictures of blender phenomena in the case of $d \neq 0$ will be presented in the next section.

\subsection{Blenders and main result}

To grasp a concept of general blender and its related properties easily, an affine example in $[5,7]$ is useful as follows. Let $h$ be a two-dimensional diffeomorphism which has an affine horseshoe for the rectangle $R=[-1,1]^{2}$ on the $x y$-plane in $\mathbb{R}^{3}$ such that

- $h(R) \cap R$ consists of two sub-rectangles $R_{1}=[-1,1] \times I_{1}$ and $R_{2}=[-1,1] \times I_{2}$, where $I_{1}, I_{2} \subset(-1,1)$ are disjoint closed intervals along the $y$-axis,

- $R \cap h^{-1}(R)$ consists of two sub-rectangles $h^{-1}\left(R_{1}\right)=J_{1} \times[-1,1]$ and $h^{-1}\left(R_{2}\right)=$ $J_{2} \times[-1,1]$ where $J_{1}, J_{2} \subset(-1,1)$ are disjoint closed intervals along the $x$-axis,

- The restriction of $h$ to $h^{-1}\left(R_{1}\right) \cup h^{-1}\left(R_{2}\right)$ is uniformly expanded and contracted along the $x$ and $y$-axis, respectively. For simplicity, the expanding ratio is supposed to be bigger than 2 .

Using the affine horseshoe map $h$, an affine blender map is defined as a diffeomorphism $\Phi: \mathbb{R}^{3} \rightarrow \mathbb{R}^{3}$ satisfying

$$
\Phi(x, y, z)= \begin{cases}(h(x, y), 5 z / 4) & \text { if }(x, y) \in h^{-1}\left(R_{1}\right) \text { and } z \in[-1,1] \\ (h(x, y), 5 z / 4-1 / 2) & \text { if }(x, y) \in h^{-1}\left(R_{2}\right) \text { and } z \in[-1,1]\end{cases}
$$

A maximal invariant set $\Lambda=\bigcap_{n \in \mathbb{Z}} \Phi^{n}(D)$, where $D=[-1,1]^{3}$ is a unit cube, is called an affine blender which has a uniformly hyperbolic splitting $T \Lambda=\mathbb{E}_{\Lambda}^{s} \oplus \mathbb{E}_{\Lambda}^{u}$ where $\mathbb{E}_{\Lambda}^{s}$ is the one-dimensional stable bundle along the $y$-axis and $\mathbb{E}_{\Lambda}^{u}$ is the two-dimensional unstable bundle along the $x z$-plane, respectively. Therefore, the index (dimension of the unstable bundle) of $\Lambda$ is equal to 2 . Set $A:=R_{1} \times[-1,1]$ and $B:=R_{2} \times[-1,3 / 4]$ of $\Phi(D) \cap D$ (see Figure 2). Denote a saddle fixed point which is contained in $A$ by $p$. Observe that the connected component of $W^{s}(p) \cap D$ containing $p$, denoted by $W_{D}^{s}(p)$, is located on the disk $D \cap\{z=0\}$. To introduce the following distinctive property with respect to stable manifold of $p$, consider an upper subset $\tilde{D}:=D \cap\{z>0\}$ of $D$ (see Figure 2). For given $y \in[-1,1], z \in(0,1)$ and $0<\varepsilon<1-z$, a two-dimensional subset $\mathcal{S}_{\varepsilon}=[-1,1] \times y \times(z, z+\varepsilon)$ in $\tilde{D}$ is called a unstable strip of width $\varepsilon$. Note that the unstable strip is tangent to $\mathbb{E}_{\Lambda}^{u}$.

Lemma 1.1.(distinctive property [3, 5, 7]) The stable manifold $W^{s}(p)$ intersects with any unstable strip $\mathcal{S}_{\varepsilon}$ of width $\varepsilon$ in $\tilde{D}$ for arbitrarily small $\varepsilon>0$. 


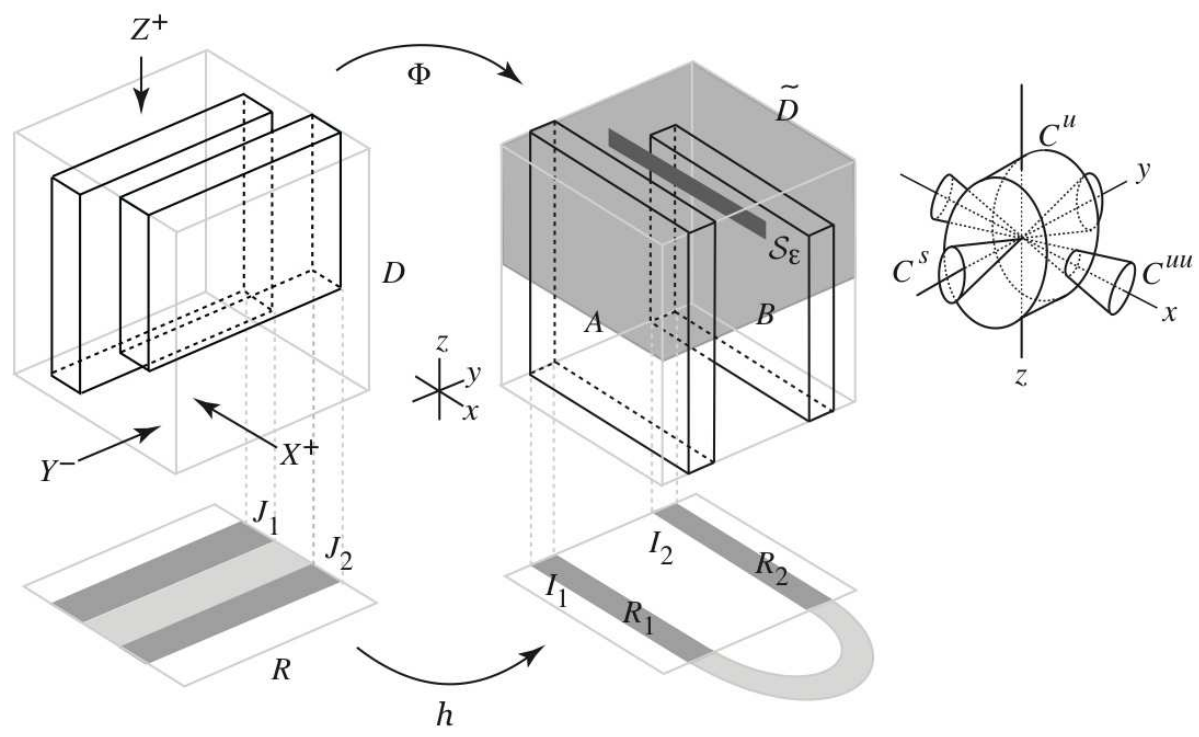

Figure 2: Affine blender map and its invariant cones

Remark 1.2. The above lemma implies that $W^{s}(p)$ is projectively dense in $\tilde{D}$, that is,

$$
\mathrm{Cl}\left(\pi_{x}\left(W^{s}(p) \cap D\right)\right) \supset \tilde{D} \cap\{x=0\},
$$

where $\mathrm{Cl}(\cdot)$ is the closure of a given set and $\pi_{x}: D \rightarrow D \cap\{x=0\}$ is a canonical projection along the strong stable direction given in the $x$-axis.

For a given unstable strip $\mathcal{S}$, let $w(\mathcal{S})$ denote the length of the shortest curve in $\mathcal{S}$ which intersects transversely with the strong unstable cone and connects the two boundary components inside the strip. Lemma 1.1 can be obtain from the following fact:

- For a given unstable strip $\mathcal{S}_{\varepsilon}$ in $\tilde{D}$, either $\Phi\left(\mathcal{S}_{\varepsilon}\right)$ intersects $W_{D}^{s}(p)$, or else $\Phi\left(\mathcal{S}_{\varepsilon}\right) \cap D$ contains a unstable strip $\mathcal{S}^{\prime}$ with $w\left(\mathcal{S}^{\prime}\right) \geq 5 \varepsilon / 4$ (see [5, Lemma 6.6] or [7]).

Now, one can extract essences from the above affine model to define general blenders from a constructive viewpoint as presented in $[5, \S 6.2 .2]$ : Let $\varphi$ be a $C^{1}$ diffeomorphism on a three-dimensional Riemannian manifold $M$ and $D \subset M$ be a copy of the threedimensional disk $[-1,1]^{3}$. We say that $(D, \varphi)$ is a blender structure or $\varphi$ has a blender $\Lambda=\bigcap_{n \in \mathbb{Z}} \varphi^{n}(D)$, if it has the following two properties:

- (hyperbolicity) there exist $\varphi$-invariant two unstable cone fields $\mathcal{C}^{u u}, \mathcal{C}^{u}$ with $\mathcal{C}^{u u} \subset$ $\mathcal{C}^{u}$ in the tangent space of $\varphi^{-1}(D) \cap D$ and $\varphi^{-1}$-invariant stable cone field $\mathcal{C}^{s}$ in the tangent space of $\varphi(D) \cap D$ such that every vector in $\mathcal{C}^{u}$ (resp. $\mathcal{C}^{s}$ ) is uniformly expanded by $d \varphi$ (resp. $d \varphi^{-1}$ ); 
- (distinctive property) for any two-dimensional strip $\mathcal{S}$ tangent to $\mathcal{C}^{u}$ crossing $D$,

- either $\varphi(\mathcal{S}) \cap D$ contains a strip $\mathcal{S}^{\prime}$ tangent to $\mathcal{C}^{u}$ which intersects the connected component of $W^{s}(p) \cap D$ containing $p$,

- or $\varphi(\mathcal{S}) \cap D$ contains a strip $\mathcal{S}^{\prime}$ with $w\left(\mathcal{S}^{\prime}\right) \geq \lambda w(\mathcal{S})$ where $\lambda>1$ is independent of $\mathcal{S}$.

Let us now state our result for the non-normally Hénon-like family on $\mathbb{R}^{3}$ defined by

$$
\varphi(x, y, z)=\varphi_{a, b, c, d}(x, y, z)=\left(1-a x^{2}+b y, x, c z+d x\right),
$$

where $a, b, c$ and $d$ are real parameters.

Main Theorem. There exists a constant $0<\delta<1 / 4$ such that if the parameters $a, b, c$, and $d$ in (1.1) satisfy the following conditions:

$$
a>a_{1}=\frac{15(1+|b|)^{2}}{4}, 0<|b|<\delta, 1+|d|<c<\frac{10}{9},|d|<\frac{1}{9},
$$

then

(1) for $d=0, \varphi_{a, b, c, 0}$ has a non-normally hyperbolic horseshoe $\Lambda_{a, b, c, 0}$ containing a saddle fixed point $p_{a, b, c, 0}$ satisfying

$$
\operatorname{dim} \operatorname{Cl}\left(\pi_{x}\left(W^{s}\left(p_{a, b, c, 0}\right)\right)=1 .\right.
$$

(2) for $d \neq 0, \varphi_{a, b, c, d}$ has a blender $\Lambda_{a, b, c, d}=\bigcap_{n \in \mathbb{Z}} \varphi_{a, b, c, d}^{n}(D)$ for some cube $D=$ $D_{a, b, c, d} \subset \mathbb{R}^{3}$, containing a saddle fixed point $p_{a, b, c, d}$ satisfying

$$
\operatorname{dim}\left(\mathrm{Cl}\left(\pi_{x}\left(W^{s}\left(p_{a, b, c, d}\right) \cap D\right)\right)\right)=2 .
$$

Remark 1.3. Numerical simulations in Figure 3 also support the main theorem. In fact, although uniformly hyperbolicity of $\varphi_{a, b, c, d}$ does not break down under conditions (1.2), geometrical dispersions of stable segments abruptly occurs if $d$ crosses 0 , which corresponds to the phase transition from the non-normally hyperbolic horseshoe to blenders.

\section{Geometrical property}

\subsection{Configurations of $\varphi$-image}

The condition (1.2) with $d \neq 0$ implies that $\varphi_{a, b, c, d}$ has a saddle fixed point of index 2 , denoted by

$$
p=p_{a, b, c, d}=\left(x_{p}, y_{p}, z_{p}\right),
$$

where

$$
x_{p}=y_{p}=\frac{(1-c) z_{p}}{d}=\frac{b-1-\sqrt{(1-b)^{2}+4 a}}{2 a} .
$$




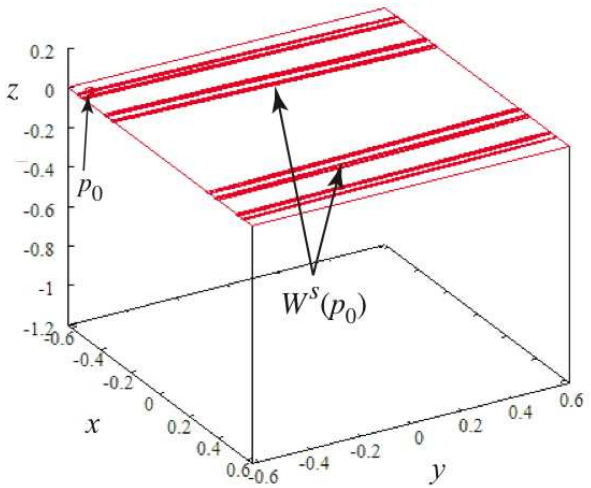

(a.1)

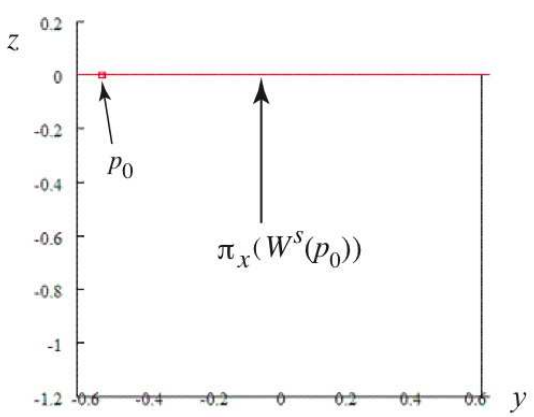

(a.2)

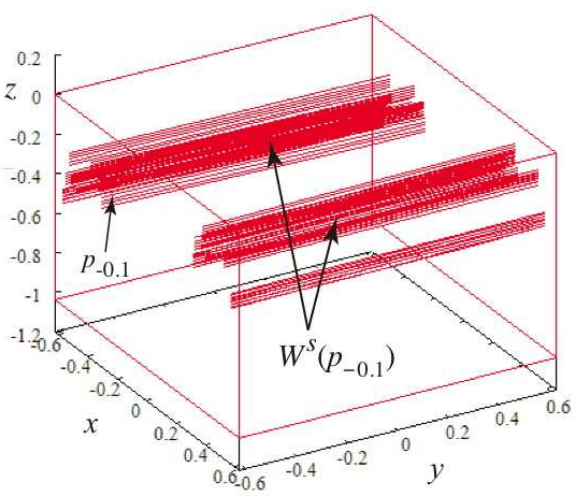

(b.1)

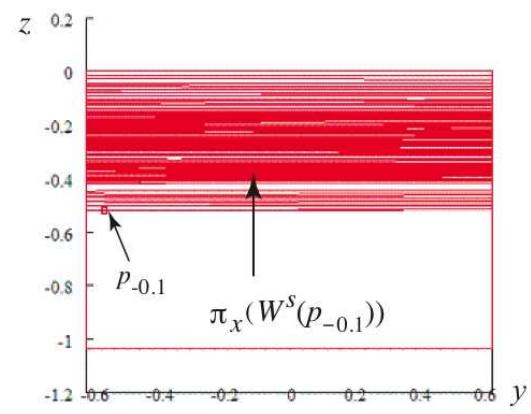

(b.2)

Figure 3: (a.1) Stable segments for $\varphi_{a, b, c, d}$ for $d=0$ and (a.2) their projective images on the $y z$-plane; (b.1) Stable segments for $\varphi_{a, b, c, d}$ for $d=-0.1$ and (b.2) their projective images on the $y z$-plane, where $(a, b, c)$ is fixed near $(5,-0.1,1.11)$.

Set a three-dimensional disk:

$$
D=D_{a, b, c, d}:=\left\{(x, y, z):|x|,|y| \leq r_{1},\left|z-z_{p}\right| \leq r_{2}\right\}
$$

where

$$
r_{1}=r_{1 ; a, b}:=\frac{3(1+|b|)+2 \sqrt{(1+|b|)^{2}+4 a}}{4 a}, \quad r_{2}=r_{2 ; a, b, c, d}:=\frac{\left|d x_{p}\right|}{c-1} .
$$

Note that

$$
\frac{|b|+1+\sqrt{(|b|+1)^{2}+4 a}}{2 a}<r_{1}<\frac{4}{5},
$$

where the second inequality is obtained from conditions for $a$ and $b$ in (1.2). Denote each side panel in $D=D_{a, b, c, d}$ by $X^{ \pm}=X_{a, b, c, d}^{ \pm}:=\left\{x= \pm r_{1}\right\} \cap D, Y^{ \pm}=Y_{a, b, c, d}^{ \pm}:=\{y=$ $\left.\pm r_{1}\right\} \cap D$, and $Z^{ \pm}=Z_{a, b, c, d}^{ \pm}:=\left\{z=z_{p} \pm r_{2}\right\} \cap D$. Remark that positions of $D_{a, b, c, d}$ and the $x y$-plane vary according to $d$, as shown in Figure 4 , satisfying as follows: 

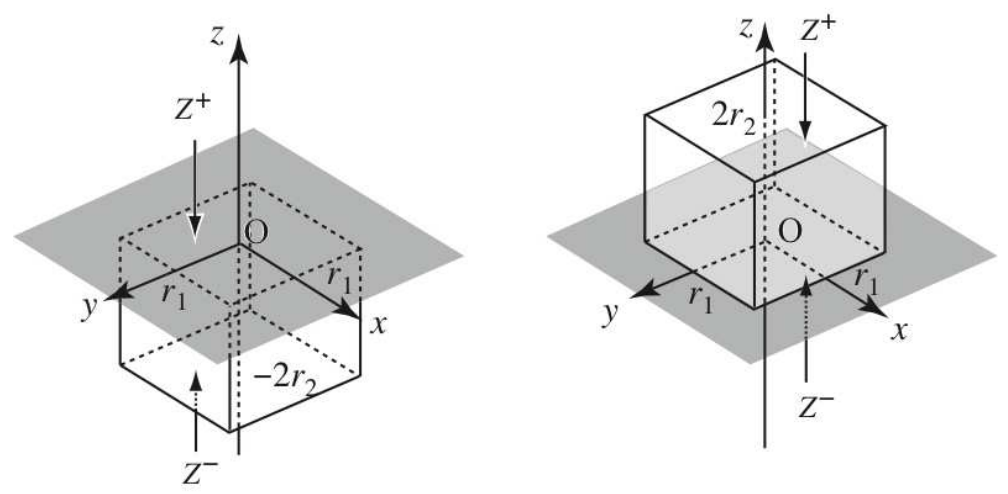

Figure 4:

- for $d<0, Z_{a, b, c, d}^{-} \subset\left\{z=-2 r_{2}\right\}$ and $Z_{a, b, c, d}^{+} \subset\{z=0\}$;

- for $d>0, Z_{a, b, c, d}^{-} \subset\{z=0\}$ and $Z_{a, b, c, d}^{+} \subset\left\{z=2 r_{2}\right\}$.

In the next proposition, we observe the positional relationship between $\varphi_{a, b, c, d}(D)$ and $D$ under the parameter condition (1.2).

Proposition 2.1. If the parameters of $\varphi=\varphi_{a, b, c, d}$ satisfy (1.2), then the intersection between $\varphi(D)$ and $D$ consists of two disjoint components $A=A_{a, b, c, d}$ and $B=B_{a, b, c, d}$ which satisfy the following conditions:

(1) If $d<0$, then

$$
A \cap\left(Y^{ \pm} \cup \varphi\left(X^{ \pm} \cup Z^{ \pm}\right)\right)=\varnothing, B \cap\left(Y^{ \pm} \cup Z^{+} \cup \varphi\left(X^{ \pm} \cup Z^{-}\right)\right)=\varnothing .
$$

(2) If $d>0$, then

$$
A \cap\left(Y^{ \pm} \cup \varphi\left(X^{ \pm} \cup Z^{ \pm}\right)\right)=\varnothing, B \cap\left(Y^{ \pm} \cup Z^{-} \cup \varphi\left(X^{ \pm} \cup Z^{+}\right)\right)=\varnothing .
$$

Proof. First, let us show the case of $d<0$. Set the points on the edges of $D$ as

$$
\left\{\begin{aligned}
P_{1} & =\left(0,-r_{1}, 0\right), & P_{2} & =\left(0,-r_{1},-2 r_{1}\right), \\
P_{1}^{ \pm} & =\left( \pm r_{1},-r_{1}, 0\right), & P_{2}^{ \pm} & =\left( \pm r_{1},-r_{1},-2 r_{1}\right), \\
Q_{1} & =\left(0, r_{1}, 0\right), & Q_{2} & =\left(0, r_{1},-2 r_{1}\right), \\
Q_{1}^{ \pm} & =\left( \pm r_{1}, r_{1}, 0\right), & Q_{2}^{ \pm} & =\left( \pm r_{1}, r_{1},-2 r_{1}\right) .
\end{aligned}\right.
$$

See Figure 5.

We here consider a canonical projection $\pi_{z}: \mathbb{R}^{3} \rightarrow \mathbb{R}^{2}$ along the $z$-axis. Since $\pi_{z} \circ \varphi_{a, b, c, d}(x, y, z)=f_{a, b}(x, y)$ which is the two-dimensional Hénon family given in the introduction, the same discussions as in [13] hold for $\pi_{z} \circ \varphi:=\pi_{z} \circ \varphi_{a, b, c, d}$, as follows: 


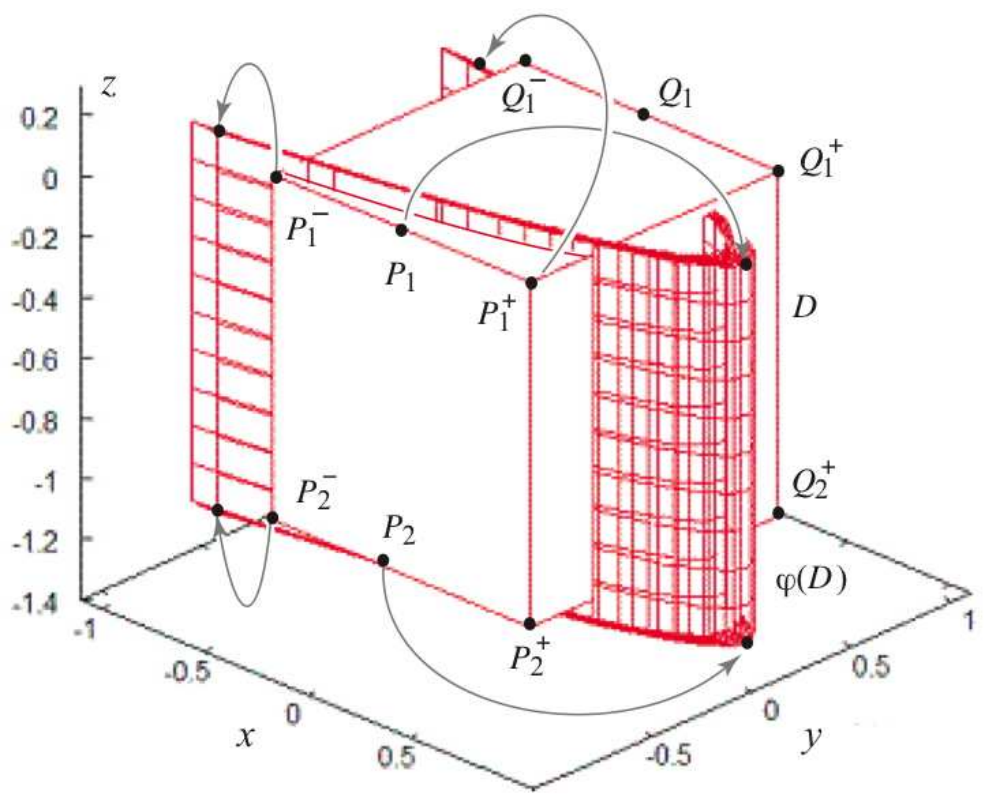

Figure 5: $D$ and $\varphi_{a, b, c, d}(D)$ when $b, d<0$.

- By $(2.2)$,

$$
1 \pm b r_{1} \geq 1-|b| r_{1}>4 / 5>r_{1} .
$$

Hence, $\pi_{z} \circ \varphi\left(P_{1}\right)=\left(1-b r_{1}, 0\right)$ and $\pi_{z} \circ \varphi\left(Q_{1}\right)=\left(1+b r_{1}, 0\right)$ are contained in the half-plane $\left\{x>r_{1}\right\}$. Also, by $(2.2)$

$$
1-a r_{1}^{2} \pm b r_{1} \leq 1-a r_{1}^{2}-|b| r_{1}<-r_{1} .
$$

Then, for each $i=1,2, \pi_{z} \circ \varphi\left(P_{i}^{-}\right)=\left(1-a r_{1}^{2}-b r_{1},-r_{1}\right), \pi_{z} \circ \varphi\left(P_{i}^{+}\right)=\left(1-a r_{1}^{2}-\right.$ $\left.b r_{1}, r_{1}\right), \pi_{z} \circ \varphi\left(Q_{i}^{-}\right)=\left(1-a r_{1}^{2}+b r_{1},-r_{1}\right)$ and $\pi_{z} \circ \varphi\left(Q_{i}^{+}\right)=\left(1-a r_{1}^{2}+b r_{1}, r_{1}\right)$ are located on $\left\{x<-r_{1}\right\}$. See Figure 6 .

- $\pi_{z} \circ \varphi\left(Y^{+}\right)$(resp. $\left.\pi_{z} \circ \varphi\left(Y^{-}\right)\right)$is a quadratic curve between $\pi_{z} \circ \varphi\left(Q_{i}^{+}\right)$and $\pi_{z} \circ \varphi\left(Q_{i}^{-}\right)$ (resp. $\pi_{z} \circ \varphi\left(P_{i}^{+}\right)$and $\pi_{z} \circ \varphi\left(P_{i}^{-}\right)$) which has the critical point $\pi_{z} \circ \varphi\left(Q_{i}\right)$ (resp. $\left.\pi_{z} \circ \varphi\left(P_{i}\right)\right)$. See Figure 6 .

Next, consider the other projection $\pi_{y}: \mathbb{R}^{3} \rightarrow \mathbb{R}^{2}$ along the $y$-axis. One can observe the following situations (see Figure 7):

- Since $\pi_{y} \circ \varphi\left(P_{1}\right)=\left(1-b r_{1}, 0\right)$ and $\pi_{y} \circ \varphi\left(P_{1}^{-}\right)=\left(1-a r_{1}^{2}-b r_{1},-d r_{1}\right)$, the subsegment of $\pi_{y} \circ \varphi\left(Z^{+}\right)$between these points has no intersection with $\pi_{y}\left(Z^{+}\right)$. On the other hand, the remaining subsegment of $\pi_{y} \circ \varphi\left(Z^{+}\right)$between $\pi_{y} \circ \varphi\left(P_{1}\right)$ and $\pi_{y} \circ \varphi\left(P_{1}^{+}\right)$has has no intersection with $\pi_{y}\left(Z^{+}\right)$, and penetrates $\pi_{y}(D)$ from $\pi_{y}\left(X^{+}\right)$to $\pi_{y}\left(X^{-}\right)$. 


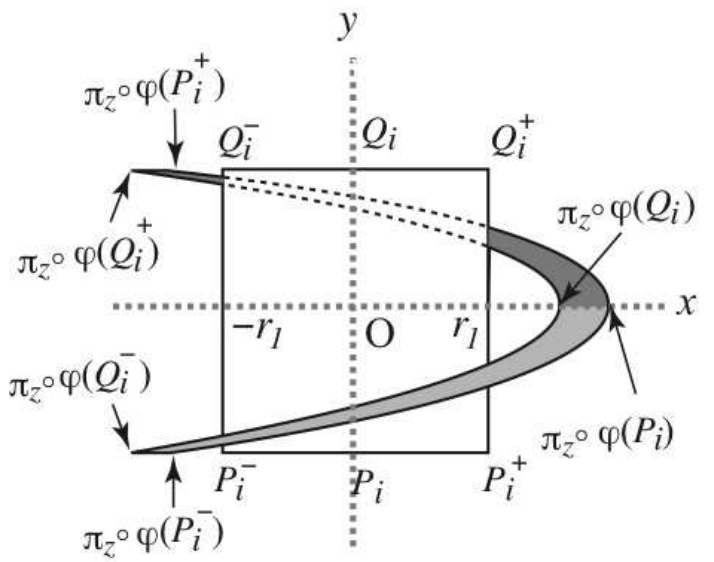

Figure 6: Projections of $D$ and $\varphi(D)$ on the $x y$-plane for $b, d<0$

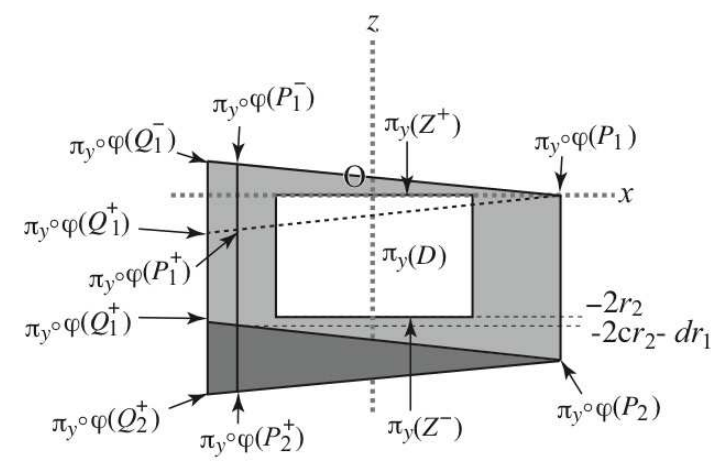

Figure 7: Projections of $D$ and $\varphi(D)$ on the $x z$-plane for $b, d<0$

- By direct calculations,

$$
-2 c r_{2}-d r_{1}<-2 r_{2} .
$$

Therefore, the subsegment of $\pi_{y} \circ \varphi\left(Z^{-}\right)$between $\pi_{y} \circ \varphi\left(P_{2}\right)=\left(1-b r_{1}, 0\right)$ and $\pi_{y} \circ \varphi\left(P_{2}^{-}\right)=\left(1-a r_{1}^{2}-b r_{1},-2 c r_{2}-d r_{1}\right)$ has no intersection with $\pi_{y}\left(Z^{-}\right)$. Also, the subsegment of $\pi_{y} \circ \varphi\left(Z^{-}\right)$between $\pi_{y} \circ \varphi\left(P_{2}\right)$ and $\pi_{y} \circ \varphi\left(P_{2}^{+}\right)$has no intersection with $\pi_{y}\left(Z^{-}\right)$.

From the above facts,

$$
A:=\varphi(D) \cap D \cap\{x<0\}, \quad B:=\varphi(D) \cap D \cap\{x>0\}
$$

satisfy the claim (1) of this proposition. One can check similarly the case (2) for $d>0$. 


\subsection{Stable cone field and structural deviation}

The next lemma is a technical result to construct cone fields assuring the uniform hyperbolicity in following several propositions. Let $A$ and $B$ be the components of $\varphi(D) \cap D$ in the above Proposition 2.1.

Lemma 2.2. Suppose that $a$ and $b$ satisfy (1.2). Then

(1) $|x|>(1+|b|) / a$ for $(x, y, z) \in \varphi^{-1}(A \cup B)$,

(2) $|y|>(1+|b|) / a$ for $(x, y, z) \in A \cup B$.

Proof. For $(x, y, z) \in \varphi^{-1}(A \cup B)$,

$$
\left|1-a x^{2}+b y\right| \leq r_{1}
$$

where $r_{1}=\left\{3(1+|b|)+2 \sqrt{(1+|b|)^{2}+4 a}\right\} /(4 a)$ given in the main theorem. Then, we have

$$
|x| \geq a^{-1 / 2} \sqrt{1-r_{1} \beta}=\frac{1}{2 a} \sqrt{4 a-\beta\left(3 \beta+2 \sqrt{\beta^{2}+4 a}\right)},
$$

where $\beta:=1+|b|$. Observe that, for every $a>15 \beta^{2} / 4$,

$$
\frac{\partial}{\partial a}\left(4 a-\beta\left(3 \beta+2 \sqrt{\beta^{2}+4 a}\right)\right)>0
$$

So, for any $a>15 \beta^{2} / 4$, we get

$$
\frac{1}{2 a} \sqrt{4 a-\beta\left(3 \beta+2 \sqrt{\beta^{2}+4 a}\right)} \geq \frac{1}{2 a} \sqrt{15 \beta^{2}-\beta\left(3 \beta+2 \sqrt{\beta^{2}+15 \beta^{2}}\right)}=\frac{\beta}{a} .
$$

That is, $|x|>(1+|b|) / a$. Moreover, one can check $|y|>(1+|b|) / a$ in essentially the same way as above.

We here construct a stable cone field $\mathcal{C}^{s}$ for which central angle is dependent on the parameter $b$. The inverse function of (1.1) is given by

$$
\varphi_{a, b, c, d}^{-1}(x, y, z)=\left(y, \frac{x-1+a y^{2}}{b}, \frac{z-d y}{c}\right) .
$$

Proposition 2.3.(stable cone fields) Let

$$
\mathcal{C}^{s}(q)=\mathcal{C}_{b}^{s}(q)=\left\{(\xi, \eta, \zeta) \in T_{q} D: \sqrt{|b|}|\eta| \geq \sqrt{\xi^{2}+\zeta^{2}}\right\}
$$

for each $q \in A \cup B$. If the parameters of $\varphi=\varphi_{a, b, c, d}$ satisfy (1.2), then each $v \in \mathcal{C}^{s}(q)$ satisfies $\left(d \varphi^{-1}\right)_{q} v \in \mathcal{C}^{s}\left(\varphi^{-1}(q)\right)$ and $\left|\left(d \varphi^{-1}\right)_{q} v\right|>\rho|v|$ for some constant $\rho>1$. 
Proof. Write $q=(x, y, z) \in A \cup B$ and $v=(\xi, \eta, \zeta) \in \mathcal{C}^{s}(q)$. From the direct calculation,

$$
u=\left(\xi_{-1}, \eta_{-1}, \zeta_{-1}\right):=\left(d \varphi^{-1}\right)_{q} v=\left(\eta, b^{-1}(\xi+2 a y \eta), c^{-1}(\zeta-d \eta)\right) .
$$

By the fact $|\eta|>\sqrt{\xi^{2}+\zeta^{2}} \geq|\xi|,|\zeta|$, and conditions $c>1,|d|<1 / 9$ in (1.2),

$$
\begin{aligned}
\xi_{-1}^{2}+\zeta_{-1}^{2} \leq & \eta^{2}+c^{-2}(|\zeta|+|d \eta|)^{2}<\eta^{2}\left\{1+c^{-2}(1+|d|)^{2}\right\} \\
& <\eta^{2}\left\{1+(1+1 / 9)^{2}\right\}=181 \eta^{2} / 81<2.3 \eta^{2}
\end{aligned}
$$

Meanwhile, by Lemma 2.2 and $0<|b|<1 / 4$,

$$
\begin{aligned}
\eta_{-1}^{2} \geq|b|^{-2}(2 a|y||\eta|-|\xi|)^{2} & \geq|b|^{-2} \eta^{2}(2 a|y|-1)^{2} \\
& \geq 4|b|^{-1} \eta^{2}\{2(1+|b|)-1\}^{2}>4|b|^{-1} \eta^{2} .
\end{aligned}
$$

Therefore, one has $|b| \eta_{-1}^{2}>\xi_{-1}^{2}+\zeta_{-1}^{2}$, that is, $u \in \mathcal{C}^{s}\left(\varphi^{-1}(q)\right)$. Moreover,

$$
\|u\|=\left|\eta_{-1}\right|>2|b|^{-1 / 2}|\eta|>4|\eta|=4\|v\|,
$$

where $\|\cdot\|$ is the maximum norm which is equivalent to the Euclidean norm.

As stated in the previous subsection, $\varphi_{a, b, c, d}(D)$ has the saddle fixed point $p$ in $A$. Denote the connected component of $W^{s}(p) \cap D$ containing $p$ by $W_{D}^{s}(p)$. From Proposition 2.1 , we have a two-dimensional subset of the boundary of $B$ which is contained in $\operatorname{Int}(D)$ for $d<0$ (resp. $d>0$ ), which is denoted by $\partial^{+} B$ (resp. $\partial^{-} B$ ), see Figure 8 .

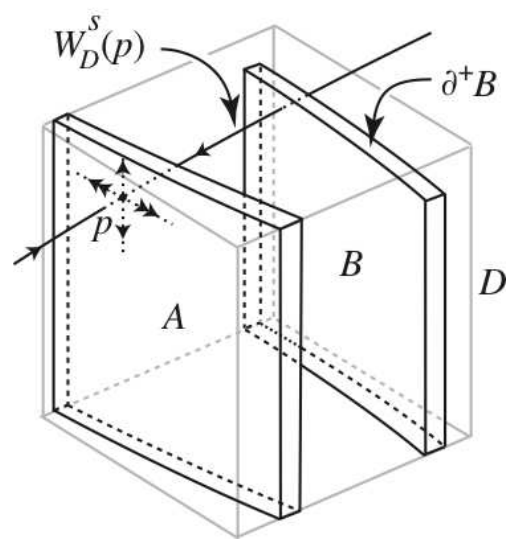

Figure 8: Positional relationship between $W_{D}^{s}(p)$ and $B$

Proposition 2.4. Suppose the parameters of $\varphi=\varphi_{a, b, c, d}$ satisfy (1.2). For any $d<0$ (resp. $d>0)$, there exist a small neighborhood $V^{+}\left(\operatorname{resp} . V^{-}\right)$of $\partial^{+} B\left(\right.$ resp. $\left.\partial^{-} B\right)$ such that

$$
V^{+} \cap W_{D}^{s}(p)=\varnothing \quad\left(\text { resp. } V^{-} \cap W_{D}^{s}(p)=\varnothing\right) .
$$


Proof. The upper bound $\delta$ for $|b|$ in the condition (1.2) will be decided definitely in this proof. For any $d<0$, observe

$$
\varphi^{-1}(B) \subset D \cap\left\{0<x \leq r_{1}\right\}
$$

Hence, for any $(x, y, z) \in \partial^{+} B$, its $z$-coordinate satisfies

$$
z \geq d r_{1}
$$

Remark that $W_{D}^{s}(p)$ is a segment through $p$, for which the tangent space is contained in the stable cone $\mathcal{C}_{b}^{s}(p)$ with opening slope is smaller than $\sqrt{|b|}$ as given in Proposition 2.3. Hence, for any $(x, y, z) \in W_{D}^{s}(p)$,

$$
z<z_{p}+2 r_{1} \sqrt{|b|}
$$

where $z_{p}$ is the $z$-coordinate of $p$. For $|b| \rightarrow 0$, one get

$$
d r_{1}=d r_{1 ; a, b}=\frac{d\left\{3(1+|b|)+2 \sqrt{(1+|b|)^{2}+4 a}\right\}}{4 a} \longrightarrow \frac{d(3+2 \sqrt{1+4 a})}{4 a}
$$

and

$$
z_{p}=z_{p_{a, b, c, d}}=\frac{d\left(b-1-\sqrt{(1-b)^{2}+4 a}\right)}{2 a(1-c)} \longrightarrow \frac{d(-1-\sqrt{1+4 a})}{2 a(1-c)} .
$$

Since $d<0$ and $c<10 / 9$,

$$
\frac{d(-1-\sqrt{1+4 a})}{2 a(1-c)}<\frac{d(3+2 \sqrt{1+4 a})}{4 a}
$$

Therefore, by taking $\delta>0$ small enough, for any $0<|b|<\delta$, one obtain

$$
z_{p_{a, b, c, d}}+2 r_{1 ; a, b} \sqrt{|b|}<d r_{1 ; a, b} .
$$

This implies that, for $d<0, W_{D}^{s}(p)$ is located below $\partial^{+} B$ as shown in Figure 8. Hence, one can take a neighborhood $V^{+}$of $\partial^{+} B$ such that $V^{+} \cap W_{D}^{s}(p)=\varnothing$.

For $d>0$, it is clear that the claim can be shown similarly. This ends the proof.

\subsection{Strong unstable and unstable cone fields}

Proposition 2.5.(unstable cone field) Let

$$
\mathcal{C}^{u}(q)=\left\{(\xi, \eta, \zeta) \in T_{q} D: \sqrt{\xi^{2}+\zeta^{2}} \geq \sqrt{2}|\eta|\right\}
$$

for any $q \in \varphi^{-1}(A \cup B)$. If the parameters of $\varphi=\varphi_{a, b, c, d}$ satisfy (1.2), then each $v \in \mathcal{C}^{u}(q)$ satisfies $(d \varphi)_{q} v \in \mathcal{C}^{u}(\varphi(q))$ and $\left|(d \varphi)_{q} v\right|>\rho|v|$ for some constant $\rho>1$. 
Proof. For any $q=(x, y, z) \in \varphi^{-1}(A \cup B)$ and $v=(\xi, \eta, \zeta) \in \mathcal{C}^{u}(q)$, set

$$
w=\left(\xi_{1}, \eta_{1}, \zeta_{1}\right):=(d \varphi)_{q} v=(-2 a x \xi+b \eta, \xi, c \zeta+d \xi)
$$

For simplicity, use the maximum norm $\|\cdot\|$ same as in the previous proof. By the condition $c>1+|d|$ in $(1.2)$,

$$
\|w\|=\max \left\{\left|\xi_{1}\right|,\left|\eta_{1}\right|,\left|\zeta_{1}\right|\right\} \geq c|\zeta|-|d||\xi|>(c-|d|)|\zeta|>|\zeta|
$$

The proof will be divided into the following three cases:

- First consider the case: $|\xi| \geq|\eta|$. By directly estimations,

$$
\begin{aligned}
\xi_{1}^{2}+\zeta_{1}^{2} \geq & \{2|\xi|(1+|b|)-|b \eta|\}^{2}+(|c \zeta|-|d \xi|)^{2} \\
& \geq\{2|\xi|(1+|b|)-|b \xi|\}^{2}>4 \xi^{2}=4 \eta_{1}^{2} .
\end{aligned}
$$

Hence, $w \in \mathcal{C}^{u}(\varphi(q))$.

If $|\xi| \geq|\zeta|$

$$
\|v\|=\max \{|\xi|,|\eta|,|\zeta|\}=|\xi|
$$

By Lemma 2.2, we have

$$
\|w\|=\max \left\{\left|\xi_{1}\right|,\left|\eta_{1}\right|,\left|\zeta_{1}\right|\right\} \geq|2 a x \xi|-|b \eta|>2|\xi|,
$$

That is, $\|w\| \geq 2\|v\|$.

On the other hand, if $|\xi|<|\zeta|$,

$$
\|v\|=\max \{|\xi|,|\eta|,|\zeta|\}=|\zeta|
$$

By (2.4), we get $\|w\| \geq \rho\|v\|$ where $\rho>1$ is some constant.

- Next, consider the case: $|\eta|>|\xi|$ and $|\zeta|>\sqrt{2}|\xi|$. By the condition $c>1+|d|$ in $(1.2)$,

$$
\xi_{1}^{2}+\zeta_{1}^{2} \geq(|c \zeta|-|d \xi|)^{2}>|\zeta|^{2}(|c|-|d|)^{2}>|\zeta|^{2}>2|\xi|^{2}=2\left|\eta_{1}\right|^{2}
$$

Then, we get $w \in \mathcal{C}^{u}(\varphi(q))$.

Since $\sqrt{2}|\eta| \leq \sqrt{\xi^{2}+\zeta^{2}}<\sqrt{\eta^{2}+\zeta^{2}}$, one can get $|\eta|<|\zeta|$ which implies that

$$
\|v\|=\max \{|\xi|,|\eta|,|\zeta|\}=|\zeta| \text {. }
$$

So, from (2.4), we obtain $\|w\| \geq \rho\|v\|$. 
- Finally, consider the remaining case: $|\eta|>|\xi|$ and $|\zeta| \leq \sqrt{2}|\xi|$. Since the previous (2.5) also holds,

$$
\sqrt{2}|\xi| \geq|\zeta|>|\eta|>|\xi|
$$

So, one can estimate as follows.

$$
\begin{aligned}
\xi_{1}^{2}+\zeta_{1}^{2} & \geq\{2|\xi|(1+|b|)-|b \eta|\}^{2}+(|c \zeta|-|d \xi|)^{2} \geq\{2|\xi|(1+|b|)-|b \xi|\}^{2} \\
& \geq\{2|\xi|(1+|b|)-\sqrt{2}|\xi||b|\}^{2}>4 \xi^{2}=4 \eta_{1}^{2} .
\end{aligned}
$$

Thus, we obtain $w \in \mathcal{C}^{u}(\varphi(q))$. Moreover, by Lemma 2.2,

$$
\|w\| \geq|-2 a x \xi+b \eta| \geq 2 a|x||\zeta|-|b||\eta|>2(1+|b|)|\zeta|-|b||\zeta|>2|\zeta|=2\|v\| .
$$

Now therefore, these cases complete the proof of this proposition.

Proposition 2.6.(strong unstable cone field) Let

$$
\mathcal{C}^{u u}(q)=\left\{(\xi, \eta, \zeta) \in T_{q} D:|\xi| \geq \sqrt{2} \sqrt{\eta^{2}+\zeta^{2}}\right\}
$$

for every $q \in \varphi^{-1}(A \cup B)$. If the parameters of $\varphi=\varphi_{a, b, c, d}$ satisfy $(1.2)$, then $(d \varphi)_{q} \mathcal{C}^{u u}(q) \subset$ $\mathcal{C}^{\text {uu }}(\varphi(q))$.

Proof. For given $q=(x, y, z) \in \varphi^{-1}(A \cup B)$ and $v=(\xi, \eta, \zeta) \in \mathcal{C}^{u u}(q)$, write

$$
\left(\xi_{1}, \eta_{1}, \zeta_{1}\right):=(d \varphi)_{q} v=(-2 a x \xi+b \eta, \xi, c \zeta+d \xi) .
$$

By Lemma 2.2 and $|\xi| / \sqrt{2} \geq|\eta|,|\zeta|$, one can obtain

$$
\xi_{1}^{2} \geq(2 a|x||\xi|-|b \eta|)^{2} \geq\{2(1+|b|)|\xi|-|b||\xi| / \sqrt{2}\}^{2} \geq 4 \xi^{2} .
$$

From the condition (1.2), observe that $|d|+c / \sqrt{2}<1$. Therefore, we get

$$
\eta_{1}^{2}+\zeta_{1}^{2} \leq \xi^{2}+(|d \xi|+c|\zeta|)^{2} \leq\left\{1+(|d|+c / \sqrt{2})^{2}\right\} \xi^{2} \leq 2 \xi^{2},
$$

which concludes $\left(\xi_{1}, \eta_{1}, \zeta_{1}\right) \in \mathcal{C}^{u u}(\varphi(q))$. This ends the proof.

\section{Proof of main result}

\subsection{Characteristic region in $D$}

We say that a segment $L$ is unstable (resp. stable) through $D$, if (i) $T_{x} L \subset \mathcal{C}^{u u}(x)$ (resp. $\mathcal{C}^{s}(x)$ ) for every $x \in L$; (ii) one of the end-points of $L$ is contained in $X^{+}$(resp. $Y^{+}$) while another is contained in $X^{-}$(resp. $Y^{-}$). Moreover, we say that an unstable segment $L^{u}$ through $D$ is in the upper (resp. lower) region to a stable segment $L^{s}$ through $D$, if $L^{u}$ dose not intersect $L^{s}$, and $L^{u}$ is in the homotopy class of the segment $L^{+}=\left[-r_{1}, r_{1}\right] \times\{0\} \times\left\{r_{2}\right\}\left(\right.$ resp. $\left.L^{-}=\left[-r_{1}, r_{1}\right] \times\{0\} \times\left\{-r_{2}\right\}\right)$ in $D \backslash L^{s}$. See Figure 9 . 


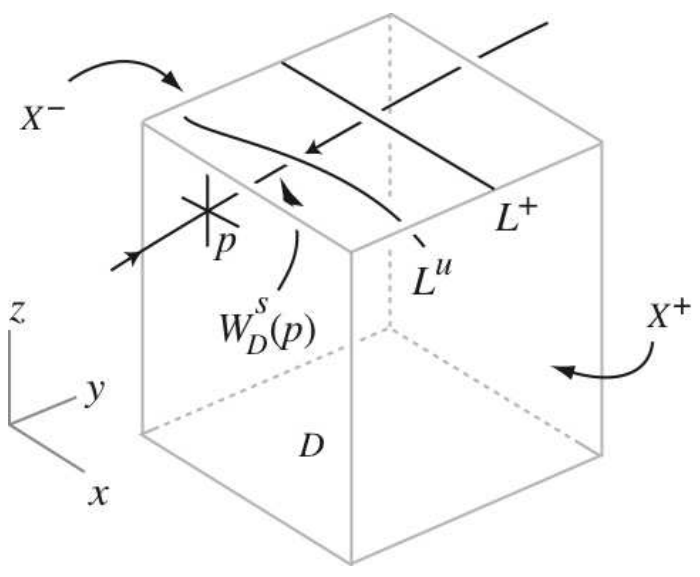

Figure 9: $L^{u}$ is located in the upper region of $W_{D}^{s}(p)$.

Proposition 3.7. Suppose the parameters of $\varphi=\varphi_{a, b, c, d}$ satisfy (1.2) with $d<0$ (resp. $d>0)$. Then, there exists a neighborhood $U^{-}\left(\right.$resp. $\left.U^{+}\right)$of $Z^{-}\left(\right.$resp. $\left.Z^{+}\right)$on the boundary of $D=D_{a, b, c, d}$ such that every unstable curve $L^{u}$ through $D$ in the upper (resp. lower) region of $W_{D}^{s}(p)$ dose not intersect $U^{-}\left(\right.$resp. $\left.U^{+}\right)$.

Proof. We here show the claim for $d<0$. In the case for $d>0$, the proof is essentially same. By $p \in \operatorname{Int}(A)$ and Proposition 2.3,

$$
\left(W_{D}^{s}(p) \cap A\right) \cap\left(Z^{ \pm} \cup X^{ \pm}\right)=\varnothing .
$$

Therefore,

$$
W_{D}^{s}(p) \subset \operatorname{Int}\left(\varphi^{-1}(A)\right) .
$$

Also, from Proposition 2.1, one can get immediately

$$
\varphi^{-1}(A) \cap\left(Z^{ \pm} \cup X^{ \pm}\right)=\varnothing .
$$

That is, it implies the existence of a sufficiently small neighborhood $U^{-}$of $Z^{-}$satisfying $U^{-} \cap \varphi^{-1}(A)=\varnothing$. Hence, one can get not only $W_{D}^{s}(p) \cap U^{-}=\varnothing$ but $L^{u} \cap U^{-}=\varnothing$ for any unstable curve $L^{u}$ in the upper region of $W_{D}^{s}(p)$.

\subsection{Sufficient condition for the blender structure}

The following properties for $\varphi_{a, b, c, d}$ will be sufficient to show the existence of blenders, which are corresponding to the conditions given primarily by Bonatti-Días [3, p.365-369].

Lemma 3.8. Suppose the parameters of $\varphi=\varphi_{a, b, c, d}$ satisfy (1.2) with $d<0$ (resp. $d>0)$. 
(I) There exists a box $D=D_{a, b, c, d} \subset \mathbb{R}^{3}$ such that $D \cap \varphi(D)$ contains a connected component $A$ disjoint from $Y^{ \pm}$and $\varphi\left(X^{ \pm} \cup Z^{ \pm}\right)$, and contains another connected component $B$ disjoint from $Y^{ \pm}, Z^{+}$and $\varphi\left(X^{ \pm} \cup Z^{-}\right)\left(\right.$resp. $\left.\varphi\left(X^{ \pm} \cup Z^{+}\right)\right)$.

(II) There exist cone fields $\mathcal{C}^{s}, \mathcal{C}^{u}, \mathcal{C}^{u u}$ and a constant $\rho>1$ satisfying as follows:

(II.a) For every $x \in A \cup B$ and every vector $v \in \mathcal{C}^{s}(x)$, the vector $w=\left(d \varphi^{-1}\right)_{x} v$ belongs to the interior of $\mathcal{C}^{s}\left(\varphi^{-1}(x)\right)$, and $|w| \geq \rho|v|$;

(II.b) For every $x \in \varphi^{-1}(A) \cup \varphi^{-1}(B)$ and every vector $v \in \mathcal{C}^{u}(x)$, the vector $w=(d \varphi)_{x} v$ belongs to the interior of $\mathcal{C}^{u}(\varphi(x))$, and $|w| \geq \rho|v|$;

(II.c) For every $x \in \varphi^{-1}(A) \cup \varphi^{-1}(B)$ and every vector $v \in \mathcal{C}^{u u}(x)$, the vector $w=(d \varphi)_{x} v$ belongs to the interior of $\mathcal{C}^{u u}(\varphi(x))$.

(III) Let $W_{\text {loc }}^{s}(p)$ be the connected component of $W^{s}(p) \cap D$ containing $p$ with index $(p)=$ 2 , which is a unique saddle fixed point for $\varphi$ in $A$. There exists a neighborhood $U^{-}$ (resp. $U^{+}$) of the side face $Z^{-}$(resp. $Z^{+}$) of $D$ such that every unstable curve $L^{u}$ through $D$ in the upper (resp. lower) region of $W_{\text {loc }}^{s}(p)$ dose not intersect $U^{-}$(resp. $\left.U^{+}\right)$.

(IV) There exist a neighborhood $U^{+}$of $Z^{+}$of $D$ and a neighborhood $V$ of $W_{\text {loc }}^{s}(p)$ such that, for every unstable curve $L^{u}$ through $D$ in the upper (resp. lower) region of $W_{\text {loc }}^{s}(p)$, one of the two following possibilities holds:

(IV.a) $\varphi(L) \cap A$ contains a unstable curve through $D$ in the upper (resp. lower) of $W_{\text {loc }}^{s}(p)$ and disjoint from $U^{+}\left(\right.$resp. $\left.U^{-}\right)$;

(IV.b) $\varphi(L) \cap B$ contains a unstable curve through $D$ in the upper (resp. lower) region of $W_{l o c}^{s}(p)$ and disjoint from $V$.

Proof. The claim (I) corresponds with Proposition 2.1, and claims in (II) are exactly same as those in Proposition 2.3, 2.5 and 2.6, respectively. Also, Proposition 3.7 implies the claim (III). Finally, by Proposition 2.4 with Proposition 2.3-2.6, we can conclude (IV) immediately

Finally, the proof of the main theorem in Subsection 1.2 is provided as follows.

Proof of Main Theorem. For $d=0$, the claim (1) of the main theorem is trivial from the observation in Subsection ??. For $d \neq 0$, since $\varphi_{a, b, c, d}$ satisfies the above geometric conditions (I) - (IV) of Lemma 3.8, from [3, p.365-369], one can directly obtain a blender $\bigcap_{n \in \mathbb{Z}} \varphi_{a, b, c, d}^{n}(D)$ as claimed in (2). The dimensional property for projected stable segments is also obtained from Lemma 1.1. This completes the proof.

\section{Acknowledgements}

The first author would like to express his appreciation to professors Bau-Sen Du, Yi-Chiuan Chen, Ming-Chia Li and Mikhail Malkin for their support and hospitality in Academia Sinica of Taiwan. 


\section{References}

[1] Z. Arai, On Hyperbolic Plateaus of the Hénon Map, Experimental Mathematics, 16:2 (2007), 181-188

[2] M. Benedicks and L. Carleson, The dynamics of the Hénon map, Ann. of Math. (2) 133 (1991), 73-169

[3] Ch. Bonatti and L. J. Díaz, Persistent Transitive Diffeomorphisms, Ann. Math. 143 (1995) 367-396

[4] Ch. Bonatti and L. J. Díaz, Robust heterodimensional cycles and $C^{1}$-generic dynamics, preprint

[5] Ch. Bonatti, L. J. Díaz, and M. Viana, Dynamics beyond uniform hyperbolicity, Encyclopaedia of Mathematical Sciences (Mathematical Physics) 102, Mathematical physics, III. Springer Verlag, 2005

[6] Y. Cao, The transverse homoclinic points are dense in the codimension-1 Hénon-like strange attractor, Proc. AMS, 127,6, Pages 1877-1883

[7] L. J. Díaz, Partial hyperbolicity, heterodimensional cycles, and transitivity, V Escuela Internacional de Sistemas Dinámicos, Facultad de Ciencias de la Universidad de Chile (January, 3-7, 2005). http://www.mat.puc-rio.br/ Iodiaz/publ.html

[8] L. J. Díaz and J. Rocha, Non-connected heterodimensional cycles: bifurcation and stability, Nonlinearity 5 (1992), $1315-1341$

[9] L. J. Díaz, Robust nonhyperbolic dynamics and heterodimensional cycles, Erg. Th. Dyn. Sys. 15(1995), 291-315

[10] L. J. Díaz and J. Rocha, Large measure of hyperbolic dynamics when unfolding heteroclinic cycles, Nonlinearity 10 (1997), 857-84.

[11] L. J. Díaz and J. Rocha, Noncritical saddle-node cycles and robust nonhyperbolic dynamics, Dynam. Stability Systems, 12 (1997), 109-135

[12] L. J. Díaz and J. Rocha, Partially hyperbolic and transitive dynamics generated by heteroclinic cycles, Ergod. Th. \& Dynam. Sys. 21(2001), 25-76

[13] R. Devaney and Z. Nitecki, Shift automorphisms in the Hénon mapping, Commun. Math. Phys., 67(1979), 137-148

[14] J. E. Fornaess and E. Gavosto, Existence of Generic Homoclinic tangencies for Hénon mappings, Journal of Geometric Analysis 2 (1992), 1-16,

[15] M. Hénon, A two dimensional mapping with a strange attractor, Comm. Math. Phys. 50 (1976), 69-77

[16] M.-C. Li and M. Malkin, Topological horseshoes for perturbations of singular difference equations, Nonlinearity, 19 (2006), 795-811

[17] L. Mora and M. Viana, Abundance of strange attractors, Acta Math. 171 (1993), 1-71

[18] J. Palis and F. Takens, Hyperbolicity and sensitive chaotic dynamics at homoclinic bifurcations. Fractal dimensions and infinitely many attractors, Cambridge Studies in Advanced Mathematics 35, Cambridge University Press, Cambridge, 1993

[19] J. Palis and M.Viana, High dimension diffeomorphisms displaying infinitely many periodic attractors, Ann. Math. 140 (1994), 207-250

[20] S. Smale, Diffeomorphisms with many periodic points, Diff. and Comb. Topology, Princeton Univ. Press (1965), 63-80. 
[21] W.-X. Qin, Chaotic invariant sets of high-dimensional Hénon-like maps, J. Math. Anal. Appl., 264(2001), 76-84

[22] M.Viana, Strange attractors in higher dimensions, Bull. Braz. Math. Soc., 24 (1993), 13-62.

Department of Mathematics, Kyoto University of Education, Fukakusa-Fujinomori 1, Fushimi, Kyoto, 612-8522, Japan.

E-mail: skiriki@kyokyo-u.ac.jp

Odessey Co. Ltd., Kasumigaseki 3-2-5, Chiyoda, Tokyo, 100-6017, Japan.

E-mail: m-nakajima@odyssey-net.jp 